\title{
The relationship between scoliosis and upper extremity functions in patients with Duchenne muscular dystrophy
}

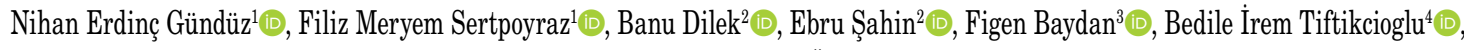

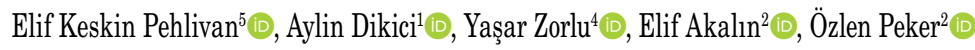 \\ ${ }^{1}$ Department of Physical Medicine and Rehabilitation, Health Science University,Izmir Tepecik Training and Research Hospital, Izmir, Turkey \\ ${ }^{2}$ Department of Physical Medicine and Rehabilitation, Dokuz Eylül University, Faculty of Medicine, Izmir, Turkey \\ ${ }^{3}$ Department of Paediatric Neurology, Health Science University, Izmir Tepecik Training and Research Hospital, Izmir, Turkey \\ ${ }^{4}$ Department of Neurology, Health Science University, Izmir Tepecik Training and Research Hospital, Izmir, Turkey \\ ${ }^{5}$ Department of Physical Medicine and Rehabilitation, Urla State Hospital, Izmir, Turkey
}

Received: June 26, 2019 Accepted: January 12, 2020 Published online: March 04, 2021

\begin{abstract}
Objectives: The aim of this study was to investigate the relationship between scoliosis and upper extremity functions in patients with Duchenne muscular dystrophy (DMD).

Patients and methods: Between January 2018 and July 2018, a total of 55 patients (54 males, 1 female; mean age: $9.9 \pm 2.9$ years; range, 6 to 15 years) who were diagnosed with DMD based on the clinical, laboratory, muscle biopsy and molecular analysis results were included in this cross-sectional study. Scoliosis was evaluated and Cobb angles were measured. Functional Ambulation Scale and Brooke and Vignos scale scores were recorded. The ABILHAND-Kids questionnaire and Nine-Hole Peg Test (9-HPT) were used to assess the upper extremity functions. Hand grip strengths were also evaluated.

Results: The median ABILHAND-Kids scores and the hand grip strength values of the patients without scoliosis were significantly higher compared to those with scoliosis ( $\mathrm{p}=0.002$ and $\mathrm{p}=0.004$ for right hand and $\mathrm{p}=0.012 \mathrm{for}$ left hand, respectively). There was no statistically significant difference in the 9-HPT scores between the patients with and without scoliosis ( $\mathrm{p}>0.05)$. We found a negative, significant correlation between the Cobb angle and ABILHAND-Kids scores in patients with scoliosis ( $\mathrm{r}=-0.503 ; \mathrm{p}=0.017)$.

Conclusion: Our study results show a moderate relationship between scoliosis and upper extremity functions. Scoliosis may adversely affect upper extremity functions in patients with DMD.
\end{abstract}

Keywords: Duchenne muscular dystrophy, grip strength, scoliosis, upper extremity function.

Duchenne muscular dystrophy (DMD), one of the most common pediatric neuromuscular diseases, is an inherited disease which affects approximately one from 5,000 live male births and displays recessive inheritance due to the $\mathrm{X}$ chromosome. ${ }^{[1]}$ Complete or partial deficiency of dystrophin among cell membrane proteins leads to progressive muscle degeneration, resulting in the loss of functional skills. ${ }^{[2]}$ Functional limitations in the lower extremities and gait disturbances are observed in the early stage, while dependence on a wheelchair may occur at the age of 12 years. Since the age of 10 years, limitations of upper extremity functions may appear. ${ }^{[3]}$ In DMD, it is of utmost importance to maintain upper extremity functions in terms of patient independence and quality of life.

\footnotetext{
Corresponding author: Nihan Erdinç Gündüz, MD. SBÜ İzmir Tepecik Eğitim ve Araştırma Hastanesi, Fiziksel Tip ve Rehabilitasyon Kliniği, 35020 Konak, İzmir, Türkiye. e-mail: nihanerdinc@yahoo.com 
Progressive muscle weakness, gait disturbances, joint contractures, cardiomyopathy, hypercapnic respiratory failure, and scoliosis are important factors affecting quality of life in patients with DMD. ${ }^{[3]}$ Scoliosis is frequently observed in patients with DMD. The time when scoliosis develops in patients with DMD coincides with the time they lose their ability to walk and stand at the age of 10 years. ${ }^{[4]}$ Although scoliosis is defined as the lateral curvature of the spine of more than 10 degrees to the right or left, which is radiologically detected on the coronal plane, indeed, it is a three-dimensional torsional deformity of the spine and body, which is not limited only on the frontal plane, together with the lateral deviation of the vertebrae. ${ }^{[5,6]}$ This deformity may be due to idiopathic, congenital or neuromuscular causes. Neuromuscular scoliosis is secondary to other pathologies, such as muscular dystrophy, cerebral palsy, spinal amyotrophy, and myelomeningocele. The Scoliosis Research Society (SRS) classifies neuromuscular scoliosis under two main headings: neuropathic causes with central and/or peripheral motor neuron involvement and myopathic causes. ${ }^{[7]}$ The incidence of scoliosis reported in DMD, which is one of the most important myopathic diseases, varies between 48 and 93\%. ${ }^{[8]}$ Neuromuscular scoliosis can progress in a severe and progressive way, and it causes more disability with the effects of the underlying disease. Oda et al. ${ }^{[9]}$ reported that scoliosis progressed continually in $46 \%$ of patients, shifted from kyphosis to lordosis in $32 \%$ of patients, and did not show progress in $15 \%$ of the patients. Brooke et al. ${ }^{[10]}$ reported that a spinal curve between $30^{\circ}$ and $120^{\circ}$ developed in $75 \%$ of 120 DMD patients. Together with muscle weakness, neuromuscular scoliosis disrupts the sitting balance in these patients, and the patients are required to use one or two hands as a support to provide this. This also restricts the patient's functional status further. ${ }^{[1,12]}$

Although the definitive treatment of DMD has not been developed yet, the life expectancy has been increased, indicating that patients spend most of their life in a wheelchair and have a life dependent on the use of the upper extremities. Therefore, it is critical to identify the factors which may affect upper extremity functions and to take precautions. To the best of our knowledge, there is no clinical study evaluating the association between the upper limb functions and scoliosis in patients with DMD. In this study, therefore, we aimed to investigate the relationship between scoliosis and upper extremity functions in patients with DMD.

\section{PATIENTS AND METHODS}

Between January 2018 and July 2018, a total of 55 patients (54 males, 1 female; mean age: 9.9 \pm 2.9 years; range, 6 to 15 years) who were diagnosed with DMD based on the clinical, laboratory, muscle biopsy and molecular analysis results and were followed at neuromuscular diseases unit were included in this cross-sectional study. Inclusion criteria were being in the age group of 6 to 15 years and having a given consent to participate in the study by themselves and/or their legal guardians. Exclusion criteria were the suspected diagnosis of DMD, the presence of an additional systemic and/or neurological disease, severe cognitive dysfunction, a history of scoliosis surgery and a history of surgical intervention for the upper extremity. A written informed consent was obtained from each patient and/or legal guardians. The study protocol was approved by the Dokuz Eylül University, Faculty of Medicine Ethics Committee (No. 2018.1.3). The study was conducted in accordance with the principles of the Declaration of Helsinki.

Demographic and clinical data including age, sex, age of diagnosis, occupation and socioeconomic status of the caregivers were obtained. Whether the participants had balance in sitting, mobilization status, the age of ending independent mobilization, use of orthopedic devices, use of a wheelchair, the age of transition to a wheelchair, the presence of contracture, participation in rehabilitation programs, and use of steroids were questioned.

Posture and scoliosis were evaluated. Scoliosis was evaluated through clinical and radiological examination. Anteroposterior and lateral scoliosis radiographs were taken for radiological evaluation. The location of scoliosis was classified as thoracic, thoracolumbar, and lumbar. The scoliosis direction was recorded as right and left. The Cobb angle measurements were performed by a radiologist. The radiographs of the participants who could not stand were taken in the sitting position. The radiographs of the participants without sitting balance were taken in the assisted sitting position.

For functional evaluation, Functional Ambulation Classification (FAC), Brooke and Vignos scales were used. The mobility status was assessed by the FAC scale, which includes the amount of physical support required to ambulate and classifies ambulation levels from degree 0 (non-functional ambulation) to degree 5 (ambulator-independent). ${ }^{[13,14]}$ The lower extremity function was assessed by the Vignos scale. The grades of the Vignos scale range from 1 to $10 ; 1$ indicates that 
the patient is able to walk and climb stairs without assistance, while Grade 10 refers to the patient being confined to a bed. ${ }^{[15]}$ The upper extremity functional levels of the participants were evaluated by the Brooke Upper Extremity Scale which was developed specifically for patients with DMD by Brooke et al. ${ }^{[16]}$ in 1981. This scale classifies functional levels from Grade 1 (defined as the ability to abduct the arms in a full circle until they touch above the head, starting with arms at the sides) to Grade 6 (defined as the inability to raise hands to the mouth and no useful function of hands). ${ }^{[16]}$

To assess the upper extremity functions, the ABILHAND-Kids questionnaire and the Nine-Hole Peg Test (9-HPT) were performed. The ABILHAND-Kids questionnaire was used to assess the manual ability. It was initially developed to measure hand functions in children with cerebral palsy. It aims to measure the children's use of their upper extremities in daily activities in 21 items. Mostly, the function of two hands together is evaluated. In general, the scale is applied by asking the caregivers, or the child can be involved, if the age is appropriate for answering questions. There should not be any assistive device or human support, when the function skill is evaluated. The participant rates the amount of difficulty during the activities on a scale from 0 to 3 . There are three points in scoring, 0 points are evaluated as cannot be done, 1 as difficult, and 2 as easy. ${ }^{[17,18]}$ The Turkish version of ABILHAND-Kids questionnaire was found to be a reliable and valid scale for children with neuromuscular diseases. ${ }^{[19]}$ The 9 -HPT is a timed test which measures finger dexterity based on performance. ${ }^{[20]}$ The patients are asked to insert the pegs that are in the box in the holes on the panel one by one and as quickly as possible, by using one hand. Then, the patient is asked to put the pegs again into the box one by one. The patient can use the other hand to fix the panel. The completion time of the test is determined by a chronometer ${ }^{[20]} \mathrm{A}$ hydraulic hand dynamometer (Jamar, North Coast Medical, CA, USA) was used to measure hand grip strength. Three measurements of muscle strength were performed for both the right and left hands, and the mean values were recorded as $\mathrm{kg}$. The participants were given a 1-min rest period between each test. ${ }^{[21]}$

\section{Statistical analysis}

The post-hoc study power analysis was performed using the $G^{*}$ Power version 3.1.9.2 software (Heinrich-Heine-Universität Düsseldorf, Düsseldorf,
Germany). Accordingly, type 1 error was taken as 0.05 , $r=-0.503$ and $n=22$, the power of the study was found to be 0.69 for the ABILHAND-Kids score.

Statistical analyses were performed using the IBM SPSS version 20.0 software (IBM Corp., Armonk, NY, USA). Descriptive data were presented in mean \pm standard deviation (SD), median (min-max) or number and frequency. The Kolmogorov-Smirnov test was used to analyze the normality of data distributions. The relationship between the Cobb angles and upper extremity function tests was evaluated using the Pearson correlation analysis $(\mathrm{r})(\mathrm{r}=0.9-1$, very high correlation; $r=0.7-0.9$, high correlation; $r=0.5-0.7$, moderate correlation; $r=0.3-0.5$, low correlation; $\mathrm{r}=0.0-0.3$ negligible correlation). ${ }^{[22]}$ Comparisons were made using the Mann-Whitney $\mathrm{U}$ test. A $p$ value of $<0.05$ was considered statistically significant.

\section{RESULTS}

Of the patients, $50.9 \%$ had independent mobilization, while $52.7 \%$ were using orthopedic devices. The rate of the patients with the FAC score of Level 0 was 47.3. The Vignos score was Level 4 and below in $56.3 \%$ of the patients. The Brooke score was Level 3 and above in $78.2 \%$ of the patients. Steroid

\begin{tabular}{|c|c|c|c|}
\hline \multicolumn{4}{|c|}{$\begin{array}{l}\text { TABLE 1 } \\
\text { Baseline demographic and clinical characteristics of } \\
\text { patients }\end{array}$} \\
\hline Variables & $\mathrm{n}$ & $\%$ & Mean \pm SD \\
\hline Age (year) & & & $9.9 \pm 2.9$ \\
\hline \multicolumn{4}{|l|}{ Sex } \\
\hline Male & 54 & 98.2 & \\
\hline Female & 1 & 1.8 & \\
\hline Age of diagnosis & & & $3.8 \pm 2.1$ \\
\hline Independent mobilization & 28 & 50.9 & \\
\hline $\begin{array}{l}\text { Age of ending independent } \\
\text { mobilization }\end{array}$ & & & $8.7 \pm 2.5$ \\
\hline Age wheelchair confined & & & $8.7 \pm 2.7$ \\
\hline \multicolumn{4}{|l|}{ Sitting balance } \\
\hline Yes & 52 & 94.5 & \\
\hline No & 3 & 5.5 & \\
\hline Orthopedic device use & 29 & 52.7 & \\
\hline \multicolumn{4}{|l|}{ Corticosteroid use } \\
\hline Yes & 21 & 38.2 & \\
\hline No & 34 & 61.8 & \\
\hline FAC level 0 & 26 & 47.3 & \\
\hline Vignos level 4 and below & 31 & 56.3 & \\
\hline Brooke level 3 and above & 43 & 78.2 & \\
\hline
\end{tabular}


use was present in 21 of 55 patients (Table 1). The dominant hand of all patients was the right hand.

A total of $33(60 \%)$ patients had no scoliosis, while $22(40 \%)$ patients had scoliosis. Four (7.3\%) patients with scoliosis had thoracic scoliosis, 10 (18.2\%) had

\begin{tabular}{|c|c|c|c|c|}
\hline \multicolumn{5}{|c|}{$\begin{array}{c}\text { TABLE } 2 \\
\text { Scoliosis measurements }\end{array}$} \\
\hline Variables & $\mathrm{n}$ & $\%$ & Median & Min-Max \\
\hline \multicolumn{5}{|l|}{ Scoliosis } \\
\hline Yes & 22 & 40 & & \\
\hline No & 33 & 60 & & \\
\hline Cobb angle & & & 17.7 & $10.80-72.00$ \\
\hline \multicolumn{5}{|c|}{$\begin{array}{l}\text { Cobb angle (according to } \\
\text { localization of scoliosis) }\end{array}$} \\
\hline Thoracic & & & 20.75 & $11.50-38.00$ \\
\hline Lumbar & & & 27.5 & $10.80-63.50$ \\
\hline Thoracolumbar & & & 13.65 & $11.00-72.00$ \\
\hline
\end{tabular}

lumbar scoliosis, and eight (14.5\%) had thoracolumbar scoliosis. The mean thoracic Cobb angle was $22.8 \pm 13.4$ degrees, the mean lumbar angle was $32.0 \pm 18.0$ degrees, and $20.7 \pm 20.9$ degrees in patients with thoracolumbar scoliosis (Table 2).

When the ABILHAND-Kids scores were evaluated according to the presence of scoliosis, the scores of patients without scoliosis were higher compared to those with scoliosis, and this difference was statistically significant $(p=0.002)$. There was no statistically significant difference between the patients with and without scoliosis in terms of 9-HPT scores ( $p>0.05)$. The hand grip strength was statistically significantly higher for both the right $(\mathrm{p}=0.004)$ and left $(\mathrm{p}=0.012)$ hands in the patients without scoliosis than those with scoliosis (Table 3). There was a negative and significant correlation between the Cobb angle and ABILHAND-Kids scores in the patients with scoliosis $(\mathrm{r}=-0.503 ; \mathrm{p}=0.017)$ (Table 4).

\begin{tabular}{|c|c|c|c|c|c|}
\hline \multirow[b]{3}{*}{ Variables } & \multicolumn{5}{|c|}{$\begin{array}{c}\text { TABLE } 3 \\
\text { Scoliosis and upper extremity functions }\end{array}$} \\
\hline & \multicolumn{2}{|c|}{ Participants with scoliosis $(n=22)$} & \multicolumn{2}{|c|}{ Participants without scoliosis $(\mathrm{n}=33$} & \multirow[b]{2}{*}{$p$} \\
\hline & Median & Min-Max & Median & Min-Max & \\
\hline ABILHAND-Kids score & 8 & $0-42$ & 34 & $7-42$ & $0.002^{\star}$ \\
\hline Nine-Hole Peg Test-remove (right hand) & 12.81 & $6-27.25$ & 11.25 & $5.40-34$ & 0.259 \\
\hline Nine-Hole Peg Test-remove (left hand) & 15.19 & $6-27.76$ & 11.10 & $5.86-30$ & 0.173 \\
\hline Nine-Hole Peg Test-place (right hand) & 20.20 & $12.61-85.7$ & 18.70 & $12-38$ & 0.317 \\
\hline Nine-Hole Peg Test-place (left hand) & 23.74 & $10-150.64$ & 21.41 & $11-38$ & 0.302 \\
\hline Hand grip strength (right hand) & 2.00 & $0-7.00$ & 5.33 & $0-16$ & $0.004^{\star}$ \\
\hline Hand grip strength (left hand) & 2.00 & $0-7.00$ & 5.10 & $0-14.33$ & $0.012^{\star}$ \\
\hline
\end{tabular}

TABLE 4
Correlations between Cobb angle and ABILHAND-Kids score, Nine-Hole Peg Test scores and
hand grip strengths ( $\mathrm{n}=22)$




\section{DISCUSSION}

In this study, we demonstrated that there was a relationship between scoliosis and upper extremity function. Upper extremity functions were negatively affected in the presence of scoliosis and associated with the Cobb angle degree in patients with DMD. To the best of our knowledge, this is the first study to investigate the relationship between scoliosis and upper extremity functions in patients with DMD.

Maintaining upper extremity functions in patients with DMD is extremely important regarding maintaining the independence and quality of life of these patients. While functional limitations in lower extremities can be compensated by the use of a wheelchair, it is difficult to compensate functional limitations in upper extremities. In a review carried out by Mazzone et al., ${ }^{[23]}$ the upper extremity muscle weaknesses of individuals with DMD started mainly in the proximal muscles, since the ambulatory periods, and proceed in a way displaying progress from proximal to distal. Movements are limited to hand and wrist and, eventually, to fingers at the non-ambulatory period. ${ }^{[23]}$ Maintaining upper extremity functions in children with DMD is of utmost importance in the continuation of independence in daily living activities, since the early period.

In the present study, upper extremity functions were evaluated using the ABILHAND-Kids questionnaire and 9-HPT, and hand grip strengths were evaluated by a dynamometer. When the ABILHAND-Kids scores were evaluated according to the presence of scoliosis, the scores of the patients without scoliosis were significantly higher compared to those with scoliosis. When the correlation between the Cobb angle and upper extremity function tests was examined, a significant negative correlation with the ABILHAND-Kids scores was found. However, no significant difference was observed in the 9-HPT results between the patients with and without scoliosis. This may be related to the fact that the 9-HPT measures finger dexterity. Fine motor skills in patients with DMD are usually deteriorated after proximal upper extremity muscle impairment. When the hand grip strengths were evaluated, the values of patients without scoliosis were higher. Muscle weakness affects the upper limb function and performance of activities of daily living. Although the relationship between the muscle strength and function is not linear, it is affected by personal and environmental factors. ${ }^{[24,25]}$

Scoliosis is progressive in the majority of patients with DMD. ${ }^{[26,27]}$ Weakness in the trunk and paraspinal muscles leads to the collapse of the immature, developing spine. ${ }^{[4]}$ Scoliosis in DMD patients progresses quite different than adolescent idiopathic scoliosis. It usually begins with a single curve with the apex in the thoracolumbar region, by progressing over time includes the entire spine and pelvic obliquity occurs. ${ }^{[28]}$ The time when scoliosis develops in patients with DMD coincides with the time they lose their ability to walk and stand at the age of 10 years. From the onset of scoliosis, progression can be extremely rapid and impair unsupported sitting ability. Scoliosis also causes deterioration in pulmonary functions. ${ }^{[29]} \mathrm{In}$ addition to all these effects of scoliosis, in this study, scoliosis was demonstrated to have an adverse effect on upper extremity functions, which was associated with the degree of scoliosis.

One of the possible mechanisms of the effect of scoliosis on upper extremity functions may be its effect on sitting balance. ${ }^{[30]}$ Severe scoliosis can cause difficulty in sitting and increases patients' need for arm support to correct the coronal balance. ${ }^{[31]}$ The correct body posture and mobility in patients with DMD are crucial in the wheelchair stage and support of upper extremity activities. Maintaining the flexibility of the spine and preventing truncal deformity provide more normal activity of upper extremities during the patient's sitting. ${ }^{[32]}$

In the study, in which Janssen et al. ${ }^{[33]}$ investigated the variables associated with upper extremity functions in patients with DMD, they found a negative correlation with the late stage of the disease, presence of scoliosis, advanced age, use of upper extremity splint, frequent constriction, limitation, and elbow pain complaints. However, they showed a positive correlation with going to school or work, use of steroids, high body mass index and advanced age at the time of diagnosis. The authors concluded that scoliosis might have an adverse effect on upper extremity functions by disrupting sitting balance.

Nonetheless, there are some limitations to this study. First, more than one test was used to evaluate the upper extremity function in this study, due to the lack of standardized tests evaluating upper extremity in neuromuscular diseases. Second, the relationship between the degree of scoliosis and upper extremity functions is complex and multifactorial. The main issue is that scoliosis would not be an independent predictor of upper extremity functions as opposed to age, steroid use, or disease progression. The lack of a sample size calculation was another limitation of this study. However, post-hoc power analysis was 
performed, and the study was found not to be powerful enough.

In conclusion, our study results show a moderate relationship between scoliosis and upper extremity functions and the presence of scoliosis in patients with DMD may adversely affect upper extremity functions. Rehabilitation interventions which prevent the development of scoliosis in these patients may contribute positively to maintenance of upper extremity functions. However, further studies investigating the relationship between scoliosis and upper extremity functions are required in this patient population.

\section{Declaration of conflicting interests}

The authors declared no conflicts of interest with respect to the authorship and/or publication of this article.

\section{Funding}

The authors received no financial support for the research and/or authorship of this article.

\section{REFERENCES}

1. Bushby K, Finkel R, Birnkrant DJ, Case LE, Clemens PR, Cripe L, et al. Diagnosis and management of Duchenne muscular dystrophy, part 1: diagnosis, and pharmacological and psychosocial management. Lancet Neurol 2010;9:77-93.

2. Bushby KM. Genetic and clinical correlations of Xp21 muscular dystrophy. J Inherit Metab Dis 1992;15:551-64.

3. Kohler M, Clarenbach CF, Bahler C, Brack T, Russi EW, Bloch KE. Disability and survival in Duchenne muscular dystrophy. J Neurol Neurosurg Psychiatry 2009;80:320-5.

4. Karol LA. Scoliosis in patients with Duchenne muscular dystrophy. J Bone Joint Surg [Am] 2007;89 Suppl 1:15562 .

5. Grivas TB, Burwell GR, Vasiliadis ES, Webb JK. A segmental radiological study of the spine and rib--cage in children with progressive infantile idiopathic scoliosis. Scoliosis 2006;1:17.

6. Grivas TB, Vasiliadis ES, Rodopoulos G. Aetiology of Idiopathic Scoliosis. What have we learned from school screening? Stud Health Technol Inform 2008;140:240-4.

7. Allam AM, Schwabe AL. Neuromuscular scoliosis. PM R 2013;5:957-63.

8. Ferrari A, Ferrara C, Balugani M, Sassi S. Severe scoliosis in neurodevelopmental disabilities: clinical signs and therapeutic proposals. Eur J Phys Rehabil Med 2010;46:563-80.

9. Oda T, Shimizu N, Yonenobu K, Ono K, Nabeshima T, Kyoh S. Longitudinal study of spinal deformity in Duchenne muscular dystrophy. J Pediatr Orthop 1993;13:478-88.

10. Brooke MH, Fenichel GM, Griggs RC, Mendell JR, Moxley R, Florence J, et al. Duchenne muscular dystrophy: patterns of clinical progression and effects of supportive therapy. Neurology 1989;39:475-81.
11. Gregoric M, Pecak F, Trontelj JV, Dimitrijević MR. Postural control in scoliosis. A statokinesimetric study in patients with scoliosis due to neuromuscular disorders and in patients with idiopathic scoliosis. Acta Orthop Scand 1981;52:59-63.

12. Berven S, Bradford DS. Neuromuscular scoliosis: causes of deformity and principles for evaluation and management. Semin Neurol 2002;22:167-78.

13. Holden MK, Gill KM, Magliozzi MR. Gait assessment for neurologically impaired patients. Standards for outcome assessment. Phys Ther 1986;66:1530-9.

14. Harris MH, Holden MK, Cahalin LP, Fitzpatrick D, Lowe S, Canavan PK. Gait in older adults: A review of the literature with an emphasis toward achieving favorable clinical outcomes, Part II. Clinical Geriatrics 2008;16:37-45.

15. Vignos PJ Jr, Spencer GE Jr, Archıbald KC. Management of progressive muscular dystrophy in childhood. JAMA 1963;184:89-96.

16. Brooke MH, Griggs RC, Mendell JR, Fenichel GM, Shumate JB, Pellegrino RJ. Clinical trial in Duchenne dystrophy. I. The design of the protocol. Muscle Nerve 1981;4:186-97.

17. Arnould C, Penta M, Renders A, Thonnard JL. ABILHANDKids: a measure of manual ability in children with cerebral palsy. Neurology 2004;63:1045-52.

18. Vandervelde L, Van den Bergh PY, Penta M, Thonnard JL. Validation of the ABILHAND questionnaire to measure manual ability in children and adults with neuromuscular disorders. J Neurol Neurosurg Psychiatry 2010;81:506-12.

19. Öksüz Ç, Alemdaroglu I, Kilinç M, Abaoğlu H, Demirci C, Karahan S, et al. Reliability and validity of the Turkish version of ABILHAND-Kids' questionnaire in a group of patients with neuromuscular disorders. Physiother Theory Pract 2017;33:780-7.

20. Smith YA, Hong E, Presson C. Normative and validation studies of the Nine-hole Peg Test with children. Percept Mot Skills 2000;90:823-43.

21. Schmidt RT, Toews JV. Grip strength as measured by the Jamar dynamometer. Arch Phys Med Rehabil 1970;51:321-7.

22. Hinkle DE, Wiersma W, Jurs SG, editors. Applied Statistics for the Behavioral Sciences. 5th ed. Boston: Houghton Mifflin; 2003.

23. Mazzone ES, Messina S, Vasco G, Main M, Eagle M, D'Amico A, et al. Reliability of the North Star Ambulatory Assessment in a multicentric setting. Neuromuscul Disord 2009;19:458-61.

24. Seferian AM, Moraux A, Annoussamy M, Canal A, Decostre V, Diebate $O$, et al. Upper limb strength and function changes during a one-year follow-up in non-ambulant patients with Duchenne Muscular Dystrophy: an observational multicenter trial. PLoS One 2015;10:e0113999.

25. Janssen MM, Bergsma A, Geurts AC, de Groot IJ. Patterns of decline in upper limb function of boys and men with DMD: an international survey. J Neurol 2014;261:1269-88.

26. Galasko CS, Williamson JB, Delaney CM. Lung function in Duchenne muscular dystrophy. Eur Spine J 1995;4:263-7.

27. Miller F, Moseley CF, Koreska J, Levison H. Pulmonary function and scoliosis in Duchenne dystrophy. J Pediatr Orthop 1988;8:133-7. 
28. Wilkins KE, Gibson DA. The patterns of spinal deformity in Duchenne muscular dystrophy. J Bone Joint Surg [Am] 1976;58:24-32.

29. Kurz LT, Mubarak SJ, Schultz P, Park SM, Leach J. Correlation of scoliosis and pulmonary function in Duchenne muscular dystrophy. J Pediatr Orthop 1983;3:347-53.

30. Flatters I, Mushtaq F, Hill LJ, Holt RJ, Wilkie RM, Mon-Williams M. The relationship between a child's postural stability and manual dexterity. Exp Brain Res 2014;232:2907-17.
31. Puddu, A, Dunn R. Neuromuscular scoliosis - surgical management and outcomes. SA Orthopaedic Journal 2013;12:53-9.

32. Duport G, Gayet E, Pries P, Thirault C, Renardel-Irani A, Fons $\mathrm{N}$, et al. Spinal deformities and wheelchair seating in Duchenne muscular dystrophy: twenty years of research and clinical experience. Semin Neurol 1995;15:29-37.

33. Janssen MM, Hendriks JC, Geurts AC, de Groot IJ. Variables associated with upper extremity function in patients with Duchenne muscular dystrophy. J Neurol 2016;263:1810-8. 